\title{
CONSTITUTIVE EQUATIONS FOR FLOW OF AN INCOMPRESSIBLE VISCOUS FLUID THROUGH A POROUS MEDIUM*
}

BY

\author{
W. O. WILLIAMS
}

Carnegie-Mellon University

Herein we present a set of equations which are proposed to describe the flow of an incompressible viscous fluid through a rigid porous medium. Terms are included which may account for capillary forces, for drag forces and for viscous shear effects. There are three unspecified coefficients in these linearized equations and we suggest how these may be evaluated, first by indicating a simple setting in which the equations reduce to D'Arcy's law, another in which they take the form of the classical diffusion equation (Fick's law) and finally by considering boundary exchange in a situation involving both free and entrapped flow. We cannot claim any substantiation of the validity of these equations. We advance them here because they seem to be the simplest elementary constitutive equations which include these three effects.

The equations arise when one seeks elementary constitutive equations within the general theory of mixtures advanced by the author and others (see [1, 2]). They are consistent with the thermodynamic theory advanced in [2]; indeed, we here present a sketch of the final reduction from the general theory in order partially to substantiate this claim. The generalization to the case in which the porous matrix is deformable may be easily found from results in [2], as may the corresponding nonlinear equations.

The application of mixture theories to the case of flow in a porous medium is a natural and obvious one, and there are a great many similar derivations in the literature: an excellent description may be found in the survey article of Atkin and Craine [3]. The justification for offering yet another consists in three points. First, and most important, the theory presented here seems to offer a minimum of complexity among this class of theories, and hence offers the best opportunity for experimental confirmation or refutation and hence for prediction. For example, we find it possible to use data from [4] to obtain estimates for the magnitude of the viscous terms in comparison with the drag terms and use these to make error estimates for measurements of relative permeability. Although some solutions have been made for previous mixture theories, it is not clear that any can be completely determined experimentally, and this presents very serious limitations on their use in practical applications.

Second, the effect of capillary forces is modeled, albeit roughly, and this leads to a reduction to the diffusion equation in certain conditions. Such a reduction, common in soil mechanics, does not appear in the general theories unless the fluid is supposed compressible.

Third, as discussed at length in [1], the general theory upon which this is based offers a

\footnotetext{
* Received November 1, 1977. The author wishes to thank M. E. Gurtin and P. A. C. Raats for criticisms of a previous draft of this article. Gurtin, in particular, pointed out that the linearized form (33) must obtain.
} 
natural interpretation for boundary forces and it is felt that this may help visualization of this difficult problem of mixture theory.

General balance of forces. In a series of papers $[1,2,6,7]$ an axiomatic foundation for a continuum theory of mixtures has been formulated. We here propose to apply the results of this theory to flow through a porous medium. Thus from the beginning we take the continuum point of view: we have a fluid body $\Theta_{f}$ and a solid body $\beta_{s}$ coincident in space, moving separately but interacting due to their coincidence. We can easily derive equations of balance of mass, which we express in the following way:

$$
\begin{aligned}
& \frac{\partial}{\partial t}\left(\rho_{s} \varphi_{s}\right)+\operatorname{div}\left(\rho_{s} \varphi_{s} v_{s}\right)=0, \\
& \frac{\partial}{\partial t}\left(\rho_{f} \varphi_{f}\right)+\operatorname{div}\left(\rho_{f} \varphi_{f} v_{f}\right)=0 .
\end{aligned}
$$

Here we assume no chemical interactions or other sources or sinks of mass. We have set $\rho_{s}$ to be the mass density of the solid making up the porous matrix, and $\varphi_{s}$ its volume ratio (volume of solid per total volume); $v_{s}$ is the velocity field of the solid. Clearly $\varphi_{s} \rho_{s}$ is the mass density of the porous medium, the body $\beta_{s}$. Similar interpretations apply to the fluid equation. By definition we must have

$$
\varphi_{s}+\varphi_{f} \leq 1 .
$$

Note that we do not assume these sum to one, and thus the saturation

$$
\varphi_{f} /\left(1-\varphi_{s}\right) \text {. }
$$

may differ from one.

The other equations which we shall consider here are the equations of balance of force. These take the following form:

$$
\begin{gathered}
\operatorname{div} T_{s}+b_{s f}+b_{s}^{e}=\rho_{s} \varphi_{s} a_{s}, \\
\operatorname{div} T_{f s}-b_{s f}-b_{f s}=0, \\
\operatorname{div} T_{f}+b_{f s}+b_{f}^{e}=\rho_{f} \varphi_{f} a_{f} .
\end{gathered}
$$

Here $T_{s}$ is the stress tensor in the porous body $\Theta_{s}$; similarly, $T_{f}$ is the stress tensor in the disperse fluid body $B_{f}$. (Thus if the fluid suffers a true internal pressure $p_{f}$ then

$$
T_{f}=-\varphi_{f} p_{f} 1
$$

where 1 is the identity tensor, for $\varphi_{f}$ measures the fraction of area contacted by the fluid.) The tensor $T_{s}$ measures the stress of the solid on itself, $T_{f}$ that of the fluid on itself; the stress $T_{f s}$ is an interaction stress, describing the forces exerted by each on the other. ${ }^{1}$ It should be noted that it is assumed that no body-couples act, so that all stresses are symmetric tensors. The term $b_{s}{ }^{e}$ denotes the external body (volume-distributed) force acting on the solid while $b_{s f}$ denotes the volume-distributed force exerted on the solid by the fluid. Finally, $a_{s}$ is the acceleration of the solid $\partial v_{s} / \partial t+\nabla v_{s} v_{s}$. Another insight into

\footnotetext{
${ }^{1}$ In [1] it is seen that if $S$ is a surface in $\bigotimes_{s}$ and $B_{f}$ then $\int_{S} T_{f s} n d a=F_{f s}(\mathcal{S})+F_{s f}(\mathcal{S})$ where $F_{f s}(\mathcal{S})$ is the contact force exerted by the solid on the fluid across $\delta$, and $F_{s}(S)$ the force exerted by the fluid on the solid across $\delta$. (It is arguable that $T_{r s}=0$ is a reasonable constitutive assumption.) Such interaction stresses, representing effects of various phases on one another, appear also in the work of Raats [5].
} 
the nature of the stresses is given by examining the boundary conditions which they satisfy. If the bodies $\beta_{s}$ and $\beta_{f}$ occupy a common region $\beta$, with boundary $\partial \beta$, then if $n$ is the exterior normal to $\partial B$,

$$
T_{s} n=\tau_{s}^{e}+\sigma_{s f}{ }^{\partial B}
$$

where $\tau_{s}{ }^{e}$ is the external force applied to the solid part of the common boundary and $\sigma_{s f}{ }^{\partial B}$ is a surface force exerted on the solid within $B$ by the fluid within $B$, e.g., a capillary force. Similarly,

$$
T_{f} n=\tau_{f}{ }^{e}+\sigma_{f s}{ }^{\partial B}
$$

while $T_{s f}$ must satisfy

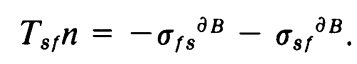

We may rearrange Eqs. (4) into the form

$$
\begin{aligned}
\operatorname{div}\left(T_{s}+\frac{1}{2} T_{f s}\right)+\frac{1}{2}\left(b_{s f}-b_{f s}\right)+b_{s}^{e} & =\rho_{s} \varphi_{s} a_{s}, \\
\operatorname{div} T_{f s}-b_{s f}-b_{s f} & =0, \\
\operatorname{div}\left(T_{f}+\frac{1}{2} T_{f s}\right)+\frac{1}{2}\left(b_{f s}-b_{s f}\right)+b_{f}^{e} & =\rho_{f} \varphi_{f} a_{f} .
\end{aligned}
$$

We choose this form in order to make it easy to use the results from [2], in which constitutive equations were written for $\frac{1}{2}\left(b_{f s}-b_{s f}\right)$ and $T_{f s}$ and $(8)_{2}$ was regarded as an identity giving the constitutive description of $b_{f s}+b_{s f} .{ }^{2}$

We now simplify the equations by supposing the porous medium to be at rest and rigid, and so allow ourselves to ignore the equations of balance of force and mass for the solid. We also suppose the fluid to be incompressible; more precisely, we suppose $\rho_{f}$ to be independent of place and time. In this way our set of equations reduces to the pair

$$
\begin{gathered}
\frac{\partial}{\partial t} \varphi+\operatorname{div}(\varphi v)=0 \\
\operatorname{div}\left(T_{f}+\frac{1}{2} T_{f s}\right)+b+b^{e}=\rho \varphi\left(\frac{\partial}{\partial t} v+\nabla v v\right) .
\end{gathered}
$$

In (9) the inessential subscripts have been dropped and $\bar{b}=\frac{1}{2}\left(b_{f s}-b_{s f}\right)$.

We remark that (9) is exactly the equation of force considered in the usual mixture theories. The difference between this approach and the traditional one lies, at this level of specialization, in the form of the constitutive equations as restricted by the ClausiusDuhem inequality.

Finally, we note that we shall have occasion to deal with the volume flux or seepage velocity

$$
q=\varphi v
$$

Constitutive equations. In order to formulate constitutive equations it is useful to introduce thermodynamic variables and thus one needs to consider also the equations of thermodynamics. In [2] this has been done for a general mixture theory and the results there have been specialized to a case of a mixture of a viscous fluid and an elastic solid. We here shall further specialize those equations.

\footnotetext{
${ }^{2}$ In [1] it is argued that $T_{s}+\frac{1}{2} T_{f s}$ and $T_{f}+\frac{1}{2} T_{f s}$ may be interpreted as the partial stresses of the traditional formulation of mixture theory.
} 
In [2] the constitutive equations begin as giving, in particular, $T_{f}, T_{f s}$ and $\tilde{b}$ as functions of the deformation of the solid, of the fluid velocity gradient, of the volume fractions of both solid and fluid, and of their gradients as well as of the thermodynamic variables. Assuming that $T_{f}$ is linear in $\nabla v$ and $\nabla \varphi$, and applying the second law of thermodynamics, one obtains (ignoring dependence of all quantities on temperature and on the presumably unvarying solid volume-fraction)

$$
\begin{gathered}
T_{f}=-p 1+2 \mu \nabla v, \\
T_{f s}=2 \tilde{p} 1, \\
\tilde{b}=\tilde{b}(v, \nabla v, \varphi, \nabla \varphi) .
\end{gathered}
$$

Here $p, \tilde{p}$ are arbitrary pressures, 1 is the identity tensor, $\mu$ is a scalar-valued function of $\varphi$, and $\nabla v$ is the symmetric part of $\nabla v .^{3}$

The residual thermodynamic restrictions on these equations are:

$$
\mu \geq 0, \quad \tilde{b} \cdot v-\beta(\varphi) \nabla \varphi \cdot v \leq 0 .^{4}
$$

Assuming that $\tilde{b}$ is linear in $v, \nabla v$ and $\nabla \varphi$, we obtain:

$$
\bar{b}=-\alpha v+\bar{\alpha} \nabla \varphi
$$

where $\alpha$ and $\bar{\alpha}$ are functions of $\varphi$. We find that $(11)_{2}$ requires

$$
\alpha \geq 0, \quad \bar{\alpha}=\beta .
$$

Then (12) and (11) complete the thermodynamic restrictions on these constitutive equations.

Under this set of assumptions the balance equations (9) become

$$
\begin{gathered}
\frac{\partial}{\partial t} \varphi+\operatorname{div}(\varphi v)=0, \\
-\nabla \bar{p}+2 \operatorname{div}(\mu \nabla v)-\alpha v+\beta \nabla \varphi+b^{e}=\rho \varphi\left(\frac{\partial}{\partial t} v+\nabla v v\right) .
\end{gathered}
$$

Here $\bar{p}$ is an indeterminant pressure. ${ }^{5}$

To proceed we need information about the material parameters $\mu, \alpha, \beta$, all of which are functions of $\varphi$. For $\mu$ a partial guide may follow from results for fluid mixtures. It appears that for mixtures of fluids

$$
\mu=\varphi^{2} \eta
$$

is appropriate; here $\eta$ is the viscosity of the base fluid and the factor $\varphi^{2}$ reflects both that $T_{f}$ is an averaged stress (across the mixture area) and that the fluid only "sees" a fraction $\varphi$ of adjacent fluid (see [8] for references). The porous matrix may present less "window" for the fluid to react on itself, or may enhance the drag, so we are led to guess

$$
\mu=\lambda \varphi^{2} \eta
$$

\footnotetext{
${ }^{3}$ To obtain (10) and (11) it is necessary to assume that the entropy flux is of the form heat flux divided by temperature, and that the term which represents the rate of working due to volume expansion is linear in div $v$.

${ }^{4} \beta(\varphi)$ is the derivative of a certain free-energy function with respect to $\varphi$; the coefficient $\gamma$ of $(10)$ is the derivative of that function with respect to solid volume-fraction.

${ }^{5}$ Thus $\dot{p}$ is to be determined by external conditions. But recall that when the medium is not saturated external conditions must include the effect of the undesignated third constituent.
} 
where $\lambda$ is a positive viscosity factor dependent only on the solid parameters, i.e. for us a constant.

Thus our equations of motion are

$$
\begin{gathered}
\frac{\partial}{\partial t} \varphi+\operatorname{div}(\varphi v)=0 \\
-\nabla \dot{p}+2 \eta \lambda \operatorname{div}\left(\varphi^{2} \widehat{\nabla v}\right)-\alpha v+\beta \nabla \varphi+b^{e}=\rho \varphi\left(\frac{\partial}{\partial t} v+\nabla v v\right) .
\end{gathered}
$$

In the succeeding sections we shall examine how one might evaluate $\alpha, \beta$ and $\lambda$.

D'Arcy's law. We first reduce the set of equations by considering a situation common to measurements of D'Arcy's law. We suppose that the flow is saturated, in a uniformly porous block of large cross-section, and that the only driving force is an applied pressure gradient. We suppose steady-state conditions have been established. Thus the viscosity term is negligible (wide cross-section), $\nabla \varphi=0, b^{e}=0$ and $a=0$. Hence

$$
-\nabla \bar{p}-\alpha v=0 \text {. }
$$

Now $\bar{p} / \varphi$ is, within a constant term, the fluid pressure applied at the ends of the section (see below), so that in the usual terms

$$
-\nabla p_{\mathrm{appl}}=-\left(\alpha / \varphi^{2}\right) q
$$

where $q$ is the volume-flux. Thus

$$
\alpha=\varphi^{2} \eta / K
$$

where $K$ is the specific permeability, as usually measured.

Fick's law. We consider a situation in which the fluid does not saturate the material and let the pressure be supposed uniform. We presume that viscous effects are negligible and that the flow is established as steady. Then

$$
0=-\alpha v+\beta \nabla \varphi
$$

Hence

$$
v=(\beta / \alpha) \nabla \varphi
$$

and the conservation of mass then requires

$$
\frac{\partial}{\partial t} \varphi+\operatorname{div}\left(\frac{\beta}{\alpha} \varphi \nabla \varphi\right)=0 .
$$

This is the equation of diffusion, and the coefficient of diffusion is

$$
D=-(\beta / \alpha) \varphi \text {. }
$$

$D$ is traditionally assumed constant, although it is now often suggested that it take the form $D_{0} \varphi^{n}$. In any case, with (16), this yields

$$
\beta=-\varphi \eta(D / K) \text {. }
$$

A situation in which $\beta$ may be measured is as shown in Fig. 1. Were $D$ constant, one would obtain

$$
\beta=\alpha v l / \pi_{s}
$$




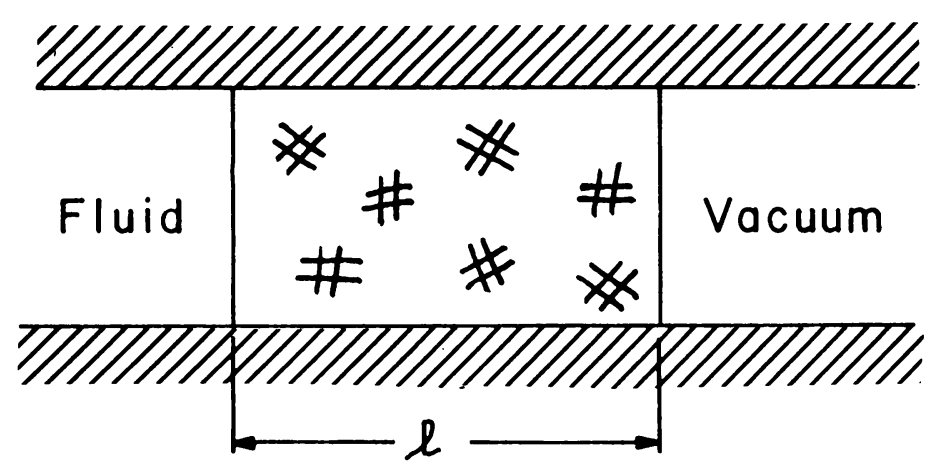

FIG. 1.

where $\pi_{s}$ is the (fixed and uniform) porosity of the medium. (In general, however, $D$ cannot be expected to be constant over such a large variation of $\varphi$.)

Boundary flow at permeable walls. Here we shall study the effect of the viscosity factor and how one might measure it. Clearly it is significant only near a boundary, but the difficulty of measuring gradients against a solid boundary rule this out as a model situation. Hence we are led to consider an open wall, that is, a case in which the porous medium terminates in a pool of the fluid. We may presume that no capillary forces are evident and we remove the likelihood of other surface affects by supposing all fluid flow is parallel the wall. Thus the significant effect is viscous shear.

In [4] Beavers and Joseph consider this problem from the opposite point of view, that of determining boundary conditions for the flow past a porous wall. ${ }^{6}$ They propose a boundary condition which ignores variation of flow through the porous medium, namely

$$
\left.\frac{d v}{d y}\right|_{0}=\frac{\gamma}{K}\left(\left.v\right|_{0}-q_{\text {ave }}\right)
$$

where the situation is as shown in Fig. 2 (a reproduction of their Fig. 1); $v$ is the velocity and $q_{\text {ave }}$ is the average volume flux through the porous medium. The flow is driven by a constant pressure gradient. They state that $\gamma$ seems to be independent of viscosity $\eta$, and

${ }^{6}$ See also [9], in which the relation of Beavers and Joseph is derived, via averaging arguments, from microscopic flow rules.

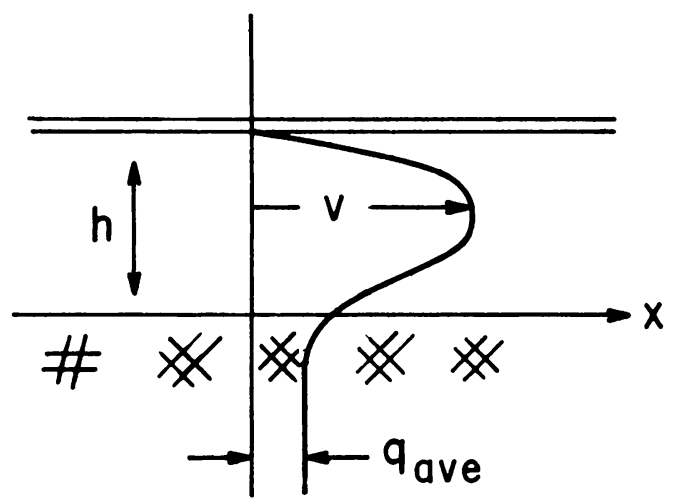

FIG. 2. 
present some experimental results, later augmented by more precise measurements in [10].

This proposal is not satisfactory to us here, as it ignores variation of velocity in the porous matrix. We shall instead solve the flow equations in both regions and devise a matching procedure as follows. On a heuristic basis we may argue first that the velocity in the free stream must be zero on the solid parts of the boundary and must match the diffusing velocity of the fluid on the "holes" in the boundary. An area average of zero velocity and the interior velocity $v$ is of course $\varphi v$, and we postulate

$$
v_{\text {outside }}=\varphi v_{\text {inside }} \text {. }
$$

Second, we suppose that the fluid and the solid each receive a shearing stress from the external stream. The part taken by the fluid is $\varphi \eta\left(\partial v_{\text {out }} / \partial n\right)$, and this must equal the force exerted by the interior fluid, $\lambda \varphi^{2} \eta(\partial v / \partial n)$; here $n$ is the normal to the surface. Hence

$$
\frac{\partial v_{\text {outside }}}{\partial n}=\lambda_{\varphi} \frac{\partial v_{\text {inside }}}{\partial n} .
$$

Thus we shall choose to match more or less according to the illustration (Fig. 2 here) of Beavers and Joseph.

In Fig. 3 we picture our model situation. Our equations are

$$
-\frac{d p}{d x}+\eta \frac{d^{2} v}{d y^{2}}=0, \quad y>0,
$$

and

$$
-\varphi \frac{d p}{d x}-\varphi^{2} \eta\left(\frac{1}{K} v-\lambda \frac{d^{2} v}{d y^{2}}\right)=0, \quad y<0 .
$$

The matching conditions are

$$
\begin{gathered}
v(0+)=\varphi v(0-), \\
\frac{d v}{d y}(0+)=\varphi \lambda \frac{d v}{d y}(0-) .
\end{gathered}
$$

The solutions we may write as

$$
\begin{aligned}
& v=V_{0}\left(h^{2}-y^{2}\right)+C(h-y), \quad y>0, \\
& v=V_{\#}+V_{\#} \frac{\sinh \delta y}{\sinh \delta l}+A \frac{\sinh \delta(y+l)}{\sinh \delta l}, \quad y<0,
\end{aligned}
$$

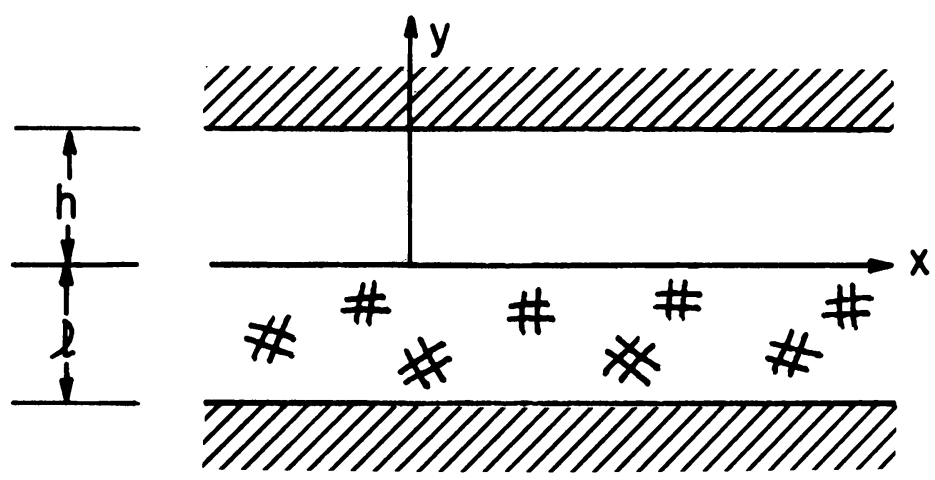

FIG. 3. 
where $A$ and $C$ are determined from (23). Here

$$
\delta=1 /(K \lambda)^{1 / 2},
$$

$V_{0}\left(h^{2}-y^{2}\right)-V_{0}(h-y)$ is the classical channel flow solution and $V_{\#}$ is the classical D'A rcy flow solution. More significant is that

$$
\frac{d v}{d y}(0+)=\lambda \delta\left\{\frac{\varphi V_{\#}}{\sinh \delta l}+\left[v(0+)-\varphi V_{\#}\right] \operatorname{coth} \delta l\right\}
$$

so that in the limit as $\delta l \rightarrow \infty$ we obtain (20):

$$
\frac{d v}{d y}(0+)=\lambda \delta\left[v(0+)-\varphi V_{\#}\right]=(\lambda / K)^{1 / 2}\left[v(0+)-\varphi V_{\#]} .\right.
$$

Thus $\lambda \delta=(\lambda / K)^{1 / 2}$ is approximately equal to the coefficient $\gamma / \sqrt{ } K$ introduced by Beavers and Joseph.

The measurements in [4] give values of $\gamma$ in the range 0.1 to 2 for various porous media, while the presumably more accurate readings of [10] give 0.1 for a particular foametal medium. Thus we may presume $0.01 \leq \lambda \leq 4$ for this class of materials. A value of $\lambda$ greater than 1 gives an augmented viscosity in the porous medium, one less than 1 a reduced viscosity.

For the purposes of measuring $\lambda$ a more direct measurement can be made, using simple viscometers. As an example we compute a simple shear solution. In Fig. 4 we diagram the model situation. We have the same geometry as before, but $v(h)=V$ and there is no pressure gradient. Solutions are

$$
\begin{gathered}
v(y)=v-\lambda \varphi \delta A(h-y), \quad y>0, \\
v(y)=A\{\sinh \delta y+\tanh \delta l \cosh \delta y\}, \quad y<0,
\end{gathered}
$$

where $A=V / \varphi(\tanh \delta l+\lambda \delta h)$. The shear force per unit area required to drive the shearing is

$$
\eta \frac{V}{h+\frac{1}{\lambda \varphi \delta} \tanh \delta l},
$$

The measurement of the shear force then yields $\lambda$. Alternatively, we can say that the viscometer with porous base has an effective width of

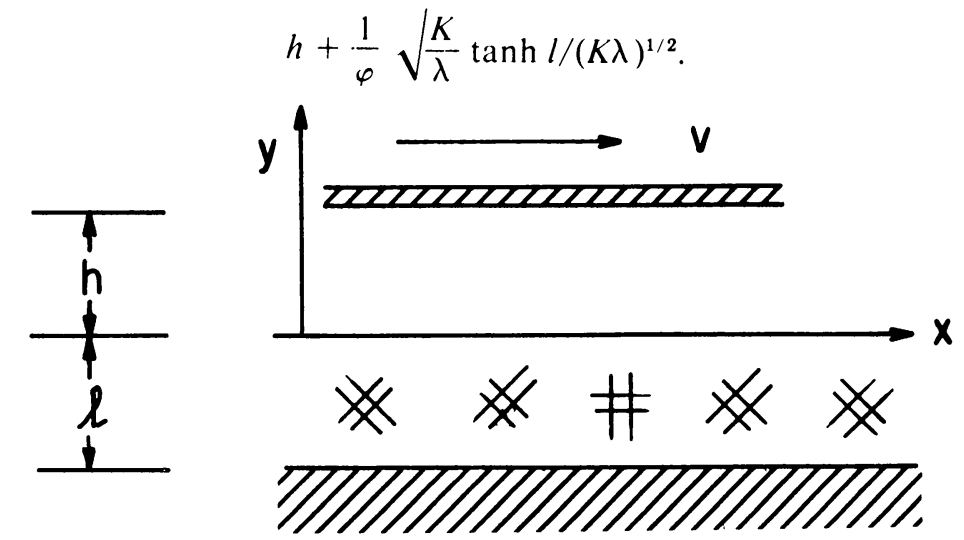

FIG. 4. 
Appropriate values (from [4]) are $K \simeq 10^{-6}$ in $^{2}$ and $\lambda \simeq 10^{-2}$, so $(K / \lambda)^{1 / 2} \simeq 10^{-2}$ in and the values of $h$ must be quite small if the effect is to be noticed.

Equations and boundary conditions. We now may regard our equations of balance as settled. Thus we have

$$
\begin{gathered}
\frac{\partial}{\partial t} \varphi+\operatorname{div}(\varphi v)=0 \\
-\nabla \bar{p}+2 \eta \lambda \operatorname{div}\left(\varphi^{2} \widehat{\nabla v}\right)-\frac{\varphi^{2} \eta}{K} v-\varphi \eta \frac{D}{K} \nabla \varphi+b^{e}=\rho \varphi\left(\frac{\partial}{\partial t} v+\nabla v v\right) .
\end{gathered}
$$

The only boundary conditions so far discussed are the defining condition

$$
\left.\left(T_{f}+\frac{1}{2} T_{f s}\right)\right|_{\text {bndry }}=\tau_{f}^{e}-\sigma
$$

where $\sigma$ is the term $\frac{1}{2}\left(\sigma_{f s}-\sigma_{s f}\right)$, and the tangential flow conditions of the last section. $\sigma$, which represents the solid-fluid interaction, can have many causes. One thinks immediately, of course, of capillary forces; there may be more esoteric effects such as surface close-packing which would create a retarding force proportional to velocity and a decrease in the viscosity window; both effects would necessarily be modeled as surface-concentrated.

Of more immediate interest are the velocity boundary conditions. First, at an impenetrable boundary we adopt a no-slip condition:

$$
v \cdot n=0
$$

where $n$ is the normal to the boundary.

Next let us consider a boundary across which there appears free fluid. Let the external velocity be $\nu_{0}$, the internal velocity $\nu_{i}$. The continuity of mass requires

$$
v_{0} \cdot n=\varphi v_{i} \cdot n
$$

and in the previous section we have argued the same should hold for the tangential flow. Hence we are led to

$$
v_{0}=\varphi v_{i}
$$

on the boundary. We have also argued in the previous section that the normal derivatives of the tangential flow are related according to a factor $\lambda_{\varphi}$ : we extend that here to suggest

$$
\frac{\partial}{\partial n} v_{0}=\lambda_{\varphi} \frac{\partial}{\partial n} v_{2} \cdot{ }^{7}
$$

It is interesting to note that (28) and the constitutive equations for the stress are in a real sense not compatible unless $\lambda=1$. We shall see that we are forced to postulate that $\sigma$ includes a shearing force exerted by the solid matrix upon the fluid.

To develop the ideas most clearly, let the surface be plane, let $n$ denote its normal; if $\nu$ is a vector let $\nu_{n}$ denote its (scalar) normal component and $\nu_{t}$ its (vectorial) tangential part,

\footnotetext{
${ }^{7} \mathrm{Eq}$. (28) states that $v_{0}$ and $q$ are equal at the boundary; here we see that the connection is not $\operatorname{smooth}\left(C^{1}\right)$. Even had we chosen to linearize $T$ in $q$ rather than in $v_{1}$ we should not have had a $C^{1}$ connection unless the viscosity coefficient was equal to one.
} 
$\partial / \partial n$ the directional derivative in the direction of $\eta$ and $\partial / \partial \tau$ the vector derivative in the plane of the surface (e.g., $\partial / \partial x \hat{\imath}+\partial / \partial y \hat{j}$ ).

The force exerted on the surface by the entrapped fluid is

$$
-\bar{p} n+\lambda \varphi^{2} \eta \nabla v_{i} n+\lambda \varphi^{2} \eta\left(\nabla v_{i}\right)^{T} n+\sigma
$$

and that exerted by the free fluid is

$$
-p n+\eta \nabla v_{0} n+\eta \nabla v_{0}^{T} n \text {. }
$$

We may argue as before that the shearing force of the free fluid should divide between solid and entrapped fluid on the basis of area average. Here this yields, in our special notation,

$$
\lambda \varphi^{2} \eta \frac{\partial v_{i \tau}}{\partial n}+\lambda \varphi^{2} \eta \frac{\partial v_{i n}}{\partial \tau}+\sigma_{\tau}=\varphi \eta \frac{\partial v_{0 \tau}}{\partial n}+\varphi \eta \frac{\partial v_{0 n}}{\partial \tau} .
$$

By using (28) and (29), this becomes

$$
\sigma_{\tau}=\varphi \eta\left[\varphi \frac{\partial v_{i n}}{\partial \tau}+v_{i n} \frac{\partial \varphi}{\partial \tau}-\lambda \varphi \frac{}{\partial \tau}\right] \partial v_{i n}
$$

Clearly it is not reasonable to suppose that $\sigma_{\tau}$ is always zero even if $\lambda=1$.

For normal forces we take account of the transport of momentum, and require the entrapped fluid to accept the fraction $\varphi$ of the external normal force plus the momentum transfer of the external fluid. Thus

$$
-\bar{p}+2 \lambda \varphi^{2} \eta \frac{\partial v_{i n}}{\partial n}+\sigma_{n}+\varphi \rho v_{i n} \frac{v_{i}{ }^{2}}{2}=-\varphi p+2 \varphi \eta \frac{\partial v_{0 n}}{\partial n}+\rho v_{0 n} \frac{v_{0}{ }^{2}}{2} .
$$

By using (28) and (29), this becomes

$$
-p+\varphi \rho v_{i n} \frac{v_{i}^{2}}{2}+\sigma_{n}=-\varphi p+\varphi^{3} \rho v_{i n} \frac{v_{i}^{2}}{2} .
$$

We will presume that $\sigma_{n}$ arises due to capillary forces or surface peculiarities of the medium. Supposing it is zero here, we shall require

$$
\bar{p}=\varphi p+\varphi\left(1-\varphi^{2}\right) \rho v_{i} \cdot n \frac{v_{i}{ }^{2}}{2} .
$$

For a porous medium terminating in a pool (28), (29) and (31) are appropriate conditions.

Finally let us return to the unsaturated situation. It is not difficult to convince oneself that the pore pressure $p_{f}$ should be constant when the medium is unsaturated. Thus the contribution to $\bar{p}$ due to $T_{f}$ would be $\varphi p_{f}$. But $p_{f}$ also acts on the solid, with a true pressure $p_{f}$ on an area fraction $\varphi$ of the solid. Thus the stress on the solid, as averaged over the solid, is $(1-\varphi) \varphi p_{f}$. Still arguing on a microscopic basis, the stress the fluid exerts on the solid is then $(1-\varphi) \varphi p_{f}$, and the stress $T_{f s}$, as defined in [1], represents the sum of the forces: $2(1-\varphi) \varphi p_{f}$. Thus we may expect that in the non-saturated cases the pressure $\bar{p}$ should take the form

$$
\begin{gathered}
\bar{p}=\varphi p_{f}-\frac{1}{2} 2(1-\varphi) \varphi p_{f} \\
\dot{p}=\varphi^{2} p_{f}
\end{gathered}
$$


where $p_{f}$ is uniform, but of arbitrary magnitude. Thus the equation of motion becomes

$$
2 \eta \lambda \operatorname{div}\left(\varphi^{2} \widehat{\nabla v}\right)-\frac{\varphi^{2} \eta}{K} v-\left(2 p_{f}+\eta \frac{D}{K}\right) \varphi \nabla \varphi+b^{e}=\rho \varphi\left(\frac{\partial}{\partial t} v+\nabla v v\right) .
$$

Boundary conditions appropriate to this case seem to be that $\varphi$ and $v$ must be given on the boundary.

Corrections to flow calculations. We have rough estimates for $\lambda$ and $K$ which should enable us to estimate the effect that the viscous drag may have on flow calculations in which viscosity is normally ignored. As an example let us take a situation which might describe a common laboratory measurement of the permeability. Consider the twodimensional flow pictured in figure 5. Assuming a constant pressure gradient through the uniform porous block, we solve

$$
P=\varphi \eta\left(\frac{v}{K}-\lambda \frac{d^{2} v}{d y^{2}}\right)
$$

to find the $x$-velocity $v$ satisfying

$$
v(h)=0, \quad \frac{d v}{d y}(0)=0 .
$$

With $V_{0}=K P / \varphi \eta$ we find

$$
v(y)=V_{0}\left(1-\frac{\cosh \delta y}{\cosh \delta h}\right)
$$

with $\delta$ as before. The average volume flux is then

$$
\begin{aligned}
\dot{q} & =V_{0 \varphi}\left(1-\frac{\tan \delta h}{\delta h}\right) \\
& =\frac{K P}{\eta}\left(1-\frac{\tanh \delta h}{\delta h}\right)
\end{aligned}
$$

and thus the usual calculation, since $P$ is the negative of the pressure gradient, gives an apparent permeability

$$
K=K\left(1-\frac{\tan \delta h}{\delta h}\right)
$$

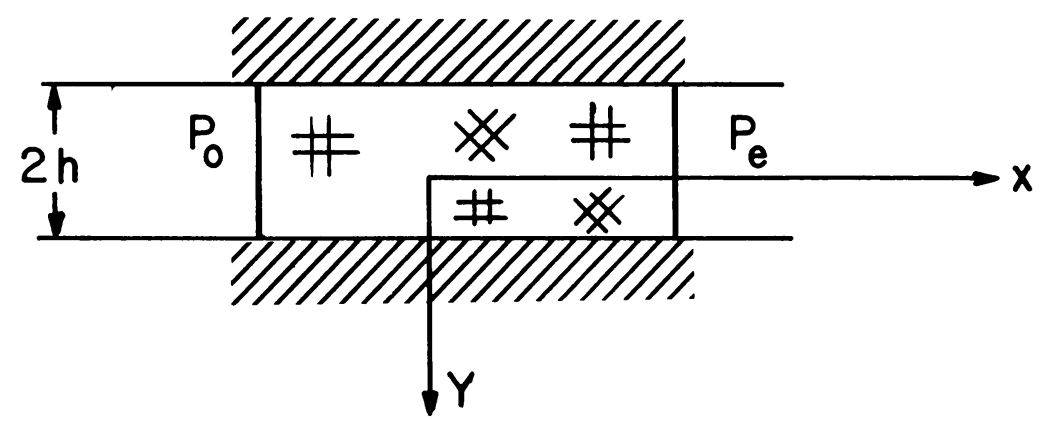

FIG. 5. 
Our estimates, $K \simeq 10^{-6} \mathrm{in}^{2}$ and $\lambda \simeq 10^{-2}$, give $\delta \sim 10^{4} \mathrm{in}^{-1}$; thus the correction to (1) is less than $1 / h 10^{-4}$ where $h$ is in inches. If $h$ is of significant size this shows that the correction due to viscosity is, as expected, small.

A modified diffusion equation. In order to reduce the equations to the classical diffusion equation we found it necessary to ignore viscous effects. Here we shall determine the form that the equations take if the terms involving viscosity are left in the system. For simplicity we restrict our attention to the case of one space dimension. We denote partial derivatives by subscripts and set

$$
\epsilon=2 \lambda K .
$$

Then if we neglect inertia and $\nabla \bar{p}^{8}$ we obtain

$$
\varphi_{t}+(\varphi v)_{x}=0, \quad \epsilon\left(\varphi^{2} v_{x}\right)_{x}-\varphi^{2} v-D \varphi \varphi_{x}=0 .
$$

Here $v$ is a scalar. We may eliminate $v$ from these equations; the result is

$$
\left(\epsilon \varphi_{x x}+\varphi\right)^{2} \varphi_{t}=\left(\epsilon \varphi \varphi_{t x}+D \varphi \varphi_{x}\right)_{x}\left(\epsilon \varphi_{x x}+\varphi\right)-\left(\epsilon \varphi \varphi_{t x}+D \varphi \varphi_{x}\right)\left(\epsilon \varphi_{x x}+\varphi\right)_{x},
$$

except where $\epsilon \varphi_{x x}+\varphi=0$; then it takes the form

$$
\epsilon \varphi \varphi_{t x}+D \varphi \varphi_{x}=0 .
$$

If the equation is now linearized about $\varphi=0$ it becomes

$$
\varphi_{t}-\epsilon \varphi_{t x x}-D \varphi_{x x}=0 .
$$

This equation occurs often in the literature. We know that $\epsilon>0$, and $D>0$ seems reasonable to assume. If so, the equation is well-behaved: Ting [11] has established a classical uniqueness result and mild minimum and maximum bounds. Barenblatt, Kochina and Zheltov [12] have found several solutions and, of particular interest here, have shown that the response to a sudden change in boundary value at $x=0$ from an initially uniform distribution shows a jump at $x=0$ which decays exponentially. Chen and Gurtin [13] have observed that (33) admits stationary discontinuities in $\varphi_{x x x}$, which decay in amplitude.

Note added in proof. I have found that the incompressibility condition was not completely exploited in [2]. For the situation considered here, it follows that in the saturated case the pressure term should have the form $-\varphi \nabla p$ rather the $-\nabla \bar{p}$. This correction to the general equation does not affect any of the calculations appearing here.

\section{REFERENCES}

[1] W. O. Williams, On the thermodynamics of mixtures, Arch. Rat. Mech. Anal. 51, 239-260 (1973)

[2] R. Sampaio and W. O. Williams, Thermodynamics of diffusing mixtures, J. Mécanique, to appear

[3] R. J. Atkin and R. E. Craine, Continuum theory of mixtures: Applications, J. Inst. Math. Appl. 17, 153-207 (1976)

[4] G. S. Beavers and D. D. Joseph, Boundary conditions at a naturally permeable wall, J. Fluid Mech. 30, 197207 (1967)

[5] P. A. C. Raats, Mechanics of saturated and unsaturated soils, to appear

[6] M. E. Gurtin and G. de la Penha, On the thermodynamics of mixtures; I. Mixtures of rigid heat conductors, Arch. Rat. Mech. Anal. 36, 390-410 (1970)

[7] M. L. Oliver, On balanced interactions in mixtures, Arch. Rat. Mech. Anal. 49, 195-224 (1972)

\footnotetext{
${ }^{8}$ Of course, since $\bar{p}=\varphi^{2}{ }_{3 /}$ we could incorporate this term directly into the diffusion term; in any case we expect the effect to be small.
} 
[8] R. Sampaio and W. O. Williams, On the viscosities of liquid mixtures, Z.A.M.P. 28, 607-614 (1977)

[9] P. G. Saffman, On the boundary conditions at the surface of a porous medium, Studies Appl. Math. 50, 93-101 (1971)

[10] G. S. Beavers, E. M. Sparrow and R. A. Magnuson, Experiments on coupled parallel flows in a channel and a bounding porous medium, J. Basic Eng., Trans. ASME, Series D, 843-848 (1970)

[11] T. W. Ting, Certain non-steady flows of second-order fluids, A rch. Rat. Mech. Anal. 14, 1-26 (1963)

[12] G. I. Barenblatt, Iu. P. Zheltov and I. N. Kochina, Basic concepts in the theory of seepage of homogeneous liquids in fissured rocks, J. Math. and Mechanics, PMM 24, 1286-1303 (1960) (transl. of Priklad. Mat. Meth. 24, 852-864 (1960))

[13] Peter J. Chen and Morton E. Gurtin, On a theory of heat conduction involving two temperatures, ZAMP 19, 614-627 (1968) 